\author{
Jean-Michel Jeannin
}

\section{Geschichtlicher Überblick}

Laut einem chinesischen Mythos entdeckte Kaiser Chen Nung, der aus hygienischen Gründen ausschliesslich heisses Wasser trank, um 2737 v. Chr. die belebende Wirkung des Tees dank einiger Blätter von Camellia sinensis, die der Wind in sein Wasser wehte. Das älteste Dokument, das die Verwendung des Tees belegt, stammt aber erst von 350 v. Chr. Im 6. Jahrhundert gelangte der Tee nach Japan. Ab 1580 führte Portugal den Tee in Europa ein. Später löste der Tee in England das Bier auf dem Frühstückstisch ab. In England wurde zur raschen Beförderung des Tees ein besonders schneller Schiffstyp, der Tee-Clipper, entwickelt. Eines dieser Boote, die Cutty Sark, kann in Greenwich besichtigt werden. Durch die Niederländer fand der Tee den Weg über den Atlantik. Im vorletzten Jahrhundert wurde in Indien C. sinensis L. var. assamica PIERRE (Assam-Tee) entdeckt und seither kultiviert [1].

\section{Camellia sinensis (L) O. KUNTZE (Theaceae)}

C. sinensis (Abb. 1) ist ein immergrüner, bis $9 \mathrm{~m}$ hoher Baum, der in Kultur auf 1-1,5 m zurückgeschnitten wird. Die weissen Blüten sind 2-3 cm gross. Die Pflanze stammt aus Südchina, Japan und Indien und wird ausser in den Ursprungsgebieten in Sri Lanka, Indonesien, Taiwan, Kenia, Uganda, in der Türkei, in den USA sowie in Südamerika, Australien und Neuseeland kultiviert. Für die Herstellung von Tee werden typischerweise die Knospen und die zwei benachbarten

\title{
Genussmittel und Ganzheitsmedizin (6)
}

Tee

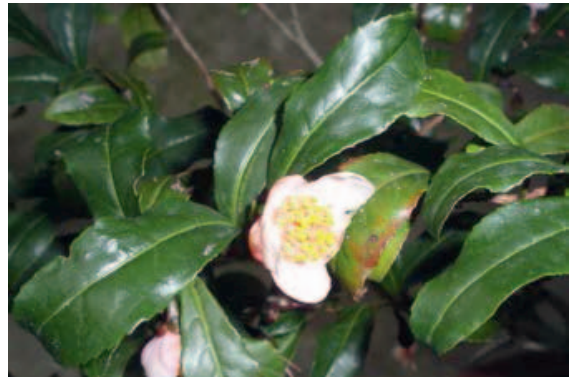

Abb. 1. Orangerosa Blüte von C. sinensis var. Beni-Bana. Botanischer Garten Basel, 2012.

Blätter verwendet [2]. Der Assam-Tee C. sinensis var. assamica unterscheidet sich hauptsächlich in der grösseren Höhe von 12 m, die die Wildpflanze erreichen kann, sowie in Einzelheiten der Inhaltsstoffe. Die Varietät wird auch als eigene Art angesehen [1].

\section{Herstellung und Zubereitung des Tees}

Zur Gewinnung von Schwarztee werden die Blätter nach dem Pflücken gerollt, eine bestimmte Zeit an der Luft gelagert und von blatteigenen Polyphenoloxidasen und Peroxidasen fermentiert. Hierbei entstehen die typische Farbe und das spezifische Aroma [3]. Die Qualitäten des Schwarztees sind international standardisiert. Man kennt vier Blattgrade: ganze Blätter, «Broken», «Fannings» und «Dust» als feinster Grad. «Tippy» heisst, es werden nur Blattspitzen verwendet; die Bezeichnung «Orange» stammt vom niederländischen Herrscherhaus Oranien und bedeutet feinste (königliche) Qualität. Zur Gewinnung von Grüntee werden die Blätter zur Inaktivierung der Enzyme geröstet (China) oder gedämpft (Japan) [1]. Der Geschmack des Grüntees hat eine herbe Note. Oolong-Tee erhält man mittels eines verkürzten Fermentationsprozesses, wozu die Blätter von Hand eingerissen statt gerollt werden. Die Herstellung von Oolong-Tee verlangt hohes handwerkliches Können. Das Ergebnis ist ein Tee mit einem feinen Geschmack und einer leichten Süsse [1]. Weisser Tee wird wie Grüntee gewonnen. Es werden jedoch nur die jüngsten Blättchen und ihre Knospen verwendet, die sich durch feine weisse Härchen auszeichnen. Man lässt die Blätter nur leicht fermentieren. Der Geschmack des weissen Tees liegt zwischen dem von Grüntee und Oolong-Tee. Schwarztee wird durch Übergiessen mit kochendem Wasser in der Kanne zubereitet. Grüntee wird mit Wasser abgegossen, das nach dem Kochen leicht abgekühlt ist (je nach Qualität des Tees bis $60^{\circ} \mathrm{C}$ ) [1].

\section{Chemie, Pharmakologie und} Toxikologie von Camellia sinensis

Tee enthält 1-5\% Koffein sowie Theobromine, Theophyllin, Dimethylxanthin, Xanthin, Adenin und weitere Xanthine, ferner 5-27\% Polyphenole (Catechin, Epigallocatechin, Epigallocatechingallat, Quercetin, Theaflavin, Thearubigin). Ein Teil des Koffeins liegt in gebundener Form vor. Weitere Inhaltsstoffe sind Fette (4-16,5\%), Aminosäuren (Grüntee > Schwarztee), Sterole und Ascorbinsäure [2], des Weiteren Theasaponin E1, Theasaponin E2 und Camellia-Saponin [4]. Die Varietät C. sinensis var. assamica enthält die spezifischen Assam-Saponine A-I [4]. Die Fermentation verändert hauptsächlich das Spektrum der Polyphenole und der Aromastoffe: Typische Bestandteile des Aromas von (c) 2012 S. Karger GmbH, Freiburg
Dipl. med. biol. Jean-Michel Jeannin Holeestrasse 43, 4054 Basel, Schweiz jm.jeannin@medwiss.ch 
Schwarztee sind Theaflavin, Thearubigin und L-Epicatechingallat [2]; das Aroma von Grüntee zeichnet sich durch Dimethylsulfoxid, Geraniol, Linalool und weitere Terpene aus [5]. Koffein stimuliert das ZNS und wirkt diuretisch [2]. Eine Tasse Grüntee enthält 24-40 mg Koffein, eine Tasse Schwarztee 14-61 mg [6]. Die Polyphenole, namentlich das Epigallocatechingallat, das im Grüntee reichlicher vorkommt als im Schwarztee, haben antikarzinogene Wirkungen [2, 7].

\section{Kulturelle Bedeutung}

Der Tee und das Teetrinken haben weltweit eine sehr grosse kulturelle Bedeutung. Der Tee war in China lange Zeit ein Kultobjekt. Er wurde in Form von Ziegeln gehandelt, von dem jeweils ein Stück abgebrochen, zerkleinert und aufgegossen wurde. In Japan wird der Tee in einer kunstvollen Zeremonie serviert, die mehrere Stunden dauern kann. In England war der Tee zuerst dem Adel und dem reichen Bürgertum vorbehalten, bevor er sich zu einem populären Getränk entwickelte. Berühmt sind der «Early Morning Tea» (Frühstückstee für einen schwungvollen Start) sowie der «Five o'Clock Tea» (Nachmittagstee als gesellschaftliches Ereignis mit Sandwiches, Hackbällchen, geräucherter Forelle und Kuchen). In Russland wird das Wasser in einem speziellen Kocher, dem Samowar, ständig heiss gehalten. Der Tee wird als sirupähnliches Konzentrat im Verhältnis

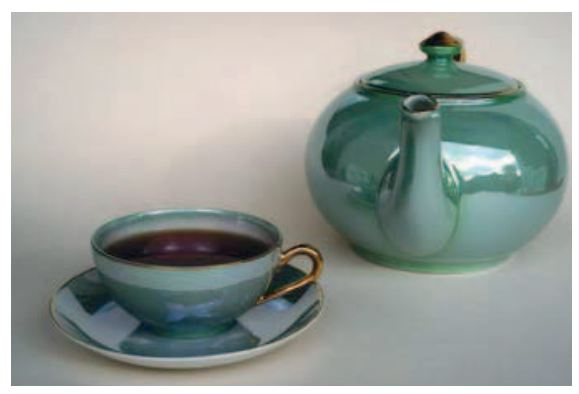

Abb. 2. Schwarztee, serviert in einem typischen Porzellanservice.
1:10 dem Wasser zugegeben. Da das Konzentrat meistens sehr stark ist, wird der Tee mit Zucker oder Konfitüre gesüsst. In Ostfriesland wird der Tee rituell gebraut, mit Kandiszucker gesüsst und kunstvoll mit Rahm versetzt. Zur Teekultur gehört auch die Verwendung besonderen Geschirrs aus Porzellan (Abb. 2) [1].

\section{Traditionelle und volksmedizinische Verwendung des Tees als Arzneimittel}

Teebeutel werden volkstümlich zum Abwaschen des Sonnenbrands, als Kompressen gegen Kopfschmerzen oder zur Behandlung von müden Augen sowie bei Zahnfleischbluten verabreicht. In China wird der Tee als Anregungsmittel, Diuretikum, Stomachikum und Expektorans sowie bei Dysenterie eingenommen. In Indien wird der Saft der Blätter zur Hämostase bei Schnitt- und anderen Verletzungen verwendet [2]. Nach Frohe [5] wird ein dünner Tee bei verdorbenem Magen, Erbrechen und Ähnlichem empfohlen. Bei Diarrhö empfiehlt sich als Adjuvans die Einnahme eines starken Teeaufgusses, der mehr Gerbstoffe enthält [5]. Regelmässige Fussbäder können bei Fusspilz helfen [1].

\section{Gesundheitlicher Nutzen des Tees}

Der regelmässige Konsum von Grüntee senkte den Gesamtcholesterolspiegel, nicht aber den Triglycerid- und den HDL-Cholesterolspiegel [8]. Der Konsum von 3 Tassen Tee täglich verminderte die Inzidenz des Herzinfarkts um 11\% [9]. Grüntee senkte die Sterblichkeit ganz allgemein, mit Ausnahme derer aufgrund von Krebs [10]. Die krebsverhütende Wirkung von Grüntee wird zwar kontrovers beurteilt, jedoch erreicht man mit dem Genuss von 3-5 Tassen Grüntee pro Tag die empfohlene tägliche Zufuhr von $250 \mathrm{mg}$ Catechinen [7].

\section{Risiken}

Personen mit bestimmten Herzrhythmusstörungen (Tachyarrhythmie), Hyperthyreose und Bluthochdruck sollten keinen (koffeinhaltigen) Tee trinken. Die Koffeinzufuhr sollte auf ca. $1 \mathrm{~g}$ pro Tag begrenzt werden.

\section{Schlussfolgerung}

Tee ist ein gesundheitlich wertvolles Genussmittel, das auch vielfältigen Genuss vermittelt. Wie der Kaffee kann der Tee jederzeit genossen werden; die intellektuelle Leistungsfähigkeit wird eher gefördert, sodass man z.B. fahrtüchtig bleibt. Das Rituelle beim Teetrinken fördert den gesellschaftlichen Zusammenhang.

\section{Literatur}

1 Haller-Zingerling C: Die Welt des Tees, ed 4. Neustadt a.d. Weinstrasse, Neuer Umschau Buchverlag GmbH, 2010.

2 Tea; in Leung AY, Foster S: Encyclopaedia of Natural Ingredients Used in Food, Drugs and Cosmetics, ed 2. New York, Wiley, 1996.

3 Engelhardt UH: Häufig gestellte Fragen zu Tee. Wissenschaftlicher Informationsdienst Tee, 2006.

4 Murakami T, et al.: Bioactive saponins and glycosides. XV. Saponin constituents with gastroprotective effect from the seeds of tea plant, Camellia sinensis L. var. assamica Pierre, cultivated in Sri Lanka: structures of assamsaponins A, B, C, D, and E. Chem Pharm Bull 1999;47:1759-1764.

5 Camellia sinensis K. O. Kuntze - Teestrauch; in Frohe D: Heilpflanzenlexikon, ed 8. Stuttgart, WVG, 2006.

6 Mayo Clinic Staff: Caffeine content for coffee, tea, soda and more. www.mayoclinic. com/health/caffeine/AN01211.

7 Boehm K, Borelli F, Ernst E, Habacher G, Hung SK, Milazzo S, Horneber M: Green tea (Camellia sinensis) for the prevention of cancer. Cochrane Database Syst Rev 2009; 3:CD005004.

$>8$ Kono S, et al: Green tea consumption and serum lipid profiles: a cross-sectional study in northern Kyushu, Japan. Prev Med 1992; 21:526-531.

9 Maron DJ: Flavonoids for reduction of atherosclerotic risk. Curr Atheroscler Rep 2004;6:73-78.

$>10$ Kuriyama S, et al: Green tea consumption and mortality due to cardiovascular disease, cancer, and all causes in Japan. JAMA 2006; 296:1255-1265. 